\title{
Christiane BAKA ОКРОВÉ, Élan vital et mystique dans la pensée d'Henri Bergson
}

Strasbourg, Presses Universitaires de Strasbourg, 2016, 295 p.

\section{Daniel Vidal}

\section{CpenEdition}

Journals

Édition électronique

URL : https://journals.openedition.org/assr/44522

DOI : $10.4000 /$ assr.44522

ISSN : 1777-5825

Éditeur

Éditions de l'EHESS

Édition imprimée

Date de publication : 1 décembre 2018

Pagination : 187-189

ISSN : 0335-5985

\section{Référence électronique}

Daniel Vidal, «Christiane вAKA окPоBÉ, Élan vital et mystique dans la pensée d'Henri Bergson », Archives de sciences sociales des religions [En ligne], 184 | octobre-décembre 2018, mis en ligne le 01 décembre 2018, consulté le 14 janvier 2022. URL : http://journals.openedition.org/assr/44522 ; DOI : https:// doi.org/10.4000/assr.44522

Ce document a été généré automatiquement le 14 janvier 2022.

(c) Archives de sciences sociales des religions 


\title{
Christiane BAKA OKPOBÉ, Élan vital et mystique dans la pensée d'Henri Bergson
}

Strasbourg, Presses Universitaires de Strasbourg, 2016, 295 p.

\author{
Daniel Vidal
}

\section{RÉFÉRENCE}

Christiane BАКА ОКРОВÉ, Élan vital et mystique dans la pensée d'Henri Bergson,

Strasbourg, Presses Universitaires de Strasbourg, 2016, 295 p.

De l'Essai sur les données immédiates de la conscience (1889) aux Deux sources de la morale et de la religion (1932) en passant par L'évolution créatrice (1907), qui forme en quelque sorte l'axe central de l'œuvre de Bergson, la thèse de l'élan vital fonde, à l'aurore du $\mathrm{xx}^{\mathrm{e}}$ siècle, une alternative et une issue décisives aux impasses et apories des philosophies mécanistes, fixistes et/ou finalistes. Les études bergsoniennes les plus récentes, de Ghislain Waterlot à François Azouvi, de Frédéric Worms à Anthony Feneuil (2013, voir Arch., 164), ont repensé la thématique de l'élan vital, et le conflit des interprétations qu'elle suscitait depuis sa proposition dans le champ philosophique. L'enjeu de ce conflit, du moins le plus important: la question de la «nature » de Dieu, " origine» « source » de cette dynamique ouverte à l'infini. Et, par là, la question de la mystique comme "raison", si l'on peut dire, de cet élan. Dans ce contexte, où se croisent les regards et s'éprouvent les plus fortes tensions, l'ouvrage de Christiane Baka Okpobé propose sans doute une des lectures les plus affinées et rigoureuses, en même temps que les plus engagées, dans la défense et illustration de la spiritualité chrétienne. Tout un travail d'écriture et d'approfondissement des textes de Bergson, toute une dérivation contrôlée des preuves et contre-preuves, placent cette réflexion parmi les plus audacieuses et les plus achevées de ses contemporaines. Pour le meilleur, ou parfois le plus contestable? Le lecteur jugera. 
2 À l'élan vital, la mystique est associée comme son signifiant dédoublé. À la fois son «accomplissement [qu'elle] porte de part en part» et son «impulsion» originelle. Mystique ne s'entend pas ici - ou pas principalement - comme relation à un Dieu vécu comme manque, et son désir d'autant plus nu, mais comme force créatrice et fondatrice, et "fine pointe de l'élan vital », alpha et oméga de l'action humaine - Dieu, pour aller à l'essentiel. Et cet essentiel s'éprouve dans la durée, expérience intime déterminante de la pensée bergsonienne du temps, et par l'intuition, «cette saisie immédiate et pleine du réel ». Expérience vécue au plus profond de la vie intérieure, qui donne accès « à l'écoulement du temps ».

3 Cette intuition de la durée permet de découvrir « dans le phénomène vital, ce par quoi il se dépasse ». Flux ouvert à l'infini, changement pur, sans cesse s'enrichissant de vitalité et de force. Vie, pour le dire en un mot, sans dessein qu'un acte d'intelligence pourrait, sinon, qualifier, et qui par là "échappe à tout contrôle ». La durée est cet "étonnement» qui, dans un flux qui s'outre-passe sans cesse, permet d'atteindre «l'absolu». Et de s'y prolonger encore. Cette durée «ne s'exprime pas en termes conceptuels ", non plus que cet élan vital, qui en est l'autre nom. Bergson insiste assez sur l'indétermination de cet élan, de cette dynamique de la vie, pour qu'on soit assuré que nul finalisme ou préfiguration, ici, ne valent. Le possible ne précède pas la réalité, comme un but à atteindre, et Bergson s'éloigne ici de Leibniz, et s'inscrit, au moins sur ce plan, dans l'héritage de Spinoza. L'évolution est ce mouvement imprévisible qui ne se clôt jamais, la matière est "de l'ordre du devenir, sans direction particulière ». La vie? «Un élan, un effort lancé à travers l'univers", une "puissance qui ne finit jamais ». Une "dilatation", écrit Jean-Louis Chrétien. Baka Okpobé en convient, et assume en ce premier temps de l'analyse, cette position philosophique capitale. Aussi bien, lorsque l'auteur définit la vie comme "de nature essentiellement spirituelle», peut-on s'étonner de ce recours soudain à une qualification trop imprécise, ou trop fortement orientée vers un a priori convenu.

4 Élan vital et élan mystique ne font, et ne sont, qu'un: "Une réfutation de la pesanteur. » Un pur vouloir, une "source d'énergie créatrice pour le moi », sans que l'on puisse, à partir de ces formulations, suspecter quelque intentionnalité. Il reste que, penser la vie, "c'est penser le Tout», et que, si l'élan vital est cette impulsion « commune aux vivants », l'homme en tant que personne advient comme « achèvement de la création ». Ou, sinon son horizon, du moins son « actualité » exacte. Sa plénitude et aussitôt sa fragilité. Sa conscience, et la « puissance de choix» dont dispose tout être humain. Mais peut-on penser l'homme comme étape ultime par quoi l'élan s'accomplit entièrement, et s'achève ? L'extrême intérêt de cet essai est d'amener le lecteur à poser la question, sans que réponse, autre que chrétienne, puisse être apportée dans le cadre interprétatif ici proposé. Il reste que l'action humaine est bien cette ouverture (aux autres) et cet affranchissement (de soi), ce "dépassement de la clôture ». Chaque moment de l'évolution, écrit Bergson, fonde " une nouveauté radicale ». La nouveauté de l'homme est sa conscience. Bien que l'auteur admette que la notion de « responsabilité ne figure pas dans le vocabulaire » du philosophe, elle dit l'homme "chargé de continuer l'œuvre de création de l'univers", lui conférant ainsi une «responsabilité », en effet, démiurgique. Jankélévitch, plus attentif à l'élan «dans l'immanence même et dans le passionnant présent", en accord plus étroit avec Bergson, écrit que "devenir est se réaliser à l'infini", loin de toute assignation à quelque mission divine. Le propre de l'homme est alors de déclore toutes les frontières, 
plus une. Il est en tel homme, disent Bergson et Gabriel Marcel, «désir d'un plusd'être ». Il est en tel homme, un élan mystique recommencé depuis le fond des choses. La mystique comme ouverture de tous les champs du possible, au-delà des choses et de leur densité matérielle, est bien, de ce point de vue, au principe de l'élan vital. De ce principe, Bergson écrit ceci, qui règle, me semble-t-il, le « problème » de Dieu : «Je parle d'un centre d'où les mondes jailliraient comme les fusées d'un immense bouquet. [Ce centre toutefois] n'est pas une chose, mais une continuité de jaillissements. Dieu ainsi défini n'a rien de tout fait ; il est vie incessante, action, liberté ».

La thèse de Bergson rencontre très vite l'opposition des penseurs catholiques, qui posent Dieu comme "être ", et transcendance, quand le philosophe dit "mouvement " et, d'une certaine façon, «immanence ». Étienne Gilson, Jacques Maritain, Joseph de Tonquedec, Maurice Blondel, autant de penseurs exigeants qui refusent que Dieu, l'élan créateur et la mystique constituent cette "réalité unique ». Contre la théorie de l'intuition comme contact avec l'immédiateté du dynamisme vital, la pensée discursive et conceptualisante, d'où toute mystique est chassée. Ce qui devait semer la consternation dans les milieux catholiques est précisément cela même qui autorise l'homme, "au-delà du donné sensible", à rechercher "la vérité de son être ", la signification de la vie et des "valeurs imprévisibles » qu'elle convoque, et qui fondent la morale. «Bien se conduire " - écrit l'auteur - "c'est savoir sans cesse se créer soimême. » Il n'est pas chez Bergson de concept d'âme au sens explicitement religieux du terme, mais comme équivalant à la notion de liberté, cet « appel qui résonne dans le moi profond », et dont "l'amour », selon Baka Okbopé, est l'accomplissement. Une telle liberté « incite le sujet à être davantage » - ce pourrait être la définition même du sujet - comme flux toujours renouvelé, impulsion toujours réamorcée, vie toujours en surcroît. La création est un acte indéfiniment recommencé, « mystique » en ceci qu'elle est ouverture effervescente à tout ce qui aspire au "désir d'absolu », qu'ainsi qualifie l'auteur. Ce désir constitue sans doute le principe de l'élan vital, où, plus qu'une " essence divine » révélée par "la vitalité de l'univers créé », s'affirme la capacité de telle énergétique à rallumer en permanence ce « feu mal éteint » que chacun (« chaque âme » selon l'auteur) porte en soi. En cela, la mystique, sans cesse transgressant l'ordre du donné, est bien ce feu originel, tel un désir inassouvi. 